\title{
PROGRAMAÇÃO DE TAREFAS EM SISTEMAS DE PRODUÇÃO PUXADA
}

\section{SCHEDULING IN PULL PRODUCTION SYSTEMS}

\author{
Patrícia Prado Belfiore ${ }^{1}$; Ciro Corrêa Leite ${ }^{2}$ \\ ${ }^{1}$ Doutoranda em Engenharia de Produção, Universidade de São Paulo, \\ Av. Prof. Almeida Prado, Trav. 2, n. 128, Cidade Universitária, São \\ Paulo - SP, CEP 05508-900, patricia.belfiore@ poli.usp.br \\ ${ }^{2}$ Professor Efetivo, Escola de Engenharia Mauá, Praça Mauá, \\ São Caetano do Sul - SP, CEP 09580-900, cirocorrea@maua.com.br
}

Recebido para publicação em: 05/10/2004

Aceito para publicação em: 25/04/2005

\section{RESUMO}

O objetivo deste trabalho é determinar a seqüência ótima de produção para um mix de produtos, de forma a permitir retiradas constantes dos processos de submontagens e suprimentos. $O$ modelo é aplicado em sistemas JIT (Just-in-time) de produção puxada. Neste sistema a produção diária é em pequena escala e variada. Assim, pode-se facilmente adaptar-se às variações de demanda e reduzir os estoques. A aplicação deste modelo leva a empresa a obter maiores lucros e melhor retorno sobre o capital investido, decorrente da redução de custos, redução de estoques e melhoria da qualidade.

Palavras-Chave: Programação de tarefas, Just in time, Produção puxada.

\section{Introdução}

Primeiramente será feita uma revisão dos conceitos de sistema JIT (just-in-time) e de produção puxada, no qual o modelo é implementado, com base nos trabalhos de BUFFA e SARIN (1987), CHASE et al. (1998) e CROSBY (1984).

O sistema JIT foi desenvolvido pela Toyota, como uma técnica de combater o desperdício. O desperdício é toda atividade que consome recursos e não agrega valor ao produto. Os estoques geram custos e ocupam espaço. Assim, uma forma de combater o desperdício, é eliminar ou reduzir ao máximo o nível de estoques.

A filosofia do sistema JIT procura produzir o componente certo, no lugar certo, e na hora certa. As partes são produzidas em tempo (just-in-time) de atenderem às necessidades de produção, o que caracteriza uma produção adaptável para atender às variações de demanda. Com a realização 
deste conceito, podem ser eliminados os estoques intermediários e de produtos acabados, e adaptarse às variações de demanda.

A aplicação adequada do sistema JIT leva a empresa a obter maiores lucros e melhor retorno sobre o capital investido, decorrente da redução de custos, redução de estoques e melhoria da qualidade.

Um dos elementos básicos do JIT é o sistema kanban, que tem como função retirar as peças em processamento de uma estação de trabalho e puxá-las para a próxima estação do processo produtivo. As partes fabricadas ou processadas são mantidas em contêineres, e somente alguns destes contêineres são fornecidos à próxima estação. Quando todos os contêineres estão cheios, a máquina pára de trabalhar, até que retorne outro contêiner vazio, que funciona como uma ordem de produção. Portanto, os estoques em processo são limitados. O programa de montagem final "puxa" as partes dos postos anteriores, e estes também puxam as partes anteriores, e assim sucessivamente, até chegar aos fornecedores externos. Nos sistemas convencionais acaba-se produzindo peças e componentes não solicitados. A fim de desocupar espaço, essa produção acaba sendo "empurrada" para o próximo posto no processo produtivo.

Outros dois elementos de um sistema JIT são: redução dos tempos de preparação das máquinas (tempo de setup), e entregas freqüentes de fornecedores, que viabilizam a produção em pequenos lotes.

No sistema JIT, o programa mestre de produção, ou programa de montagem final, tem um horizonte de 1 a 3 meses. No mês corrente, o programa mestre é balanceado em bases diárias, a fim de garantir carga uniforme para as máquinas e para os fornecedores.

Em um sistema de produção puxada, procura-se manter constante o nível de estoques entre as estações. As necessidades de produção, tanto mensal quanto diária, são constantes. O controle das ordens de produção é feito pelo kanban.

Peças são produzidas em processos de submontagens para a montagem do produto final. $\mathrm{O}$ objetivo do modelo é determinar a sequiência ótima do mix de produção, de forma que cada peça tenha uma velocidade de consumo constante. Mantendo constante a velocidade de retirada das peças de cada processo, pode-se eliminar ou reduzir os estoques intermediários (em processo) e os de produtos acabados, e adaptar a produção às variações de demanda.

Na seção 2 será feita uma descrição do problema de programação de tarefas em uma indústria automobilística. A seção 3 apresenta a modelagem matemática do sistema. A seção 4 descreve um estudo de caso real com aplicação do modelo desenvolvido. Finalmente no item 5 estão as considerações finais. 


\section{Descrição do problema}

O modelo, que será descrito na seção 3, é aplicado em uma montadora multinacional, que atua nos setores automotivos, de transporte e serviços, e trabalha com produção puxada e sistema JIT.

O processo de planejamento da montadora segue o modelo clássico, em nível estratégico, tático e operacional (longo, médio e curto prazo). O planejamento estratégico é feito para um período de 5 anos e consiste em investimentos de longo prazo da empresa. O planejamento de médio prazo é feito para um horizonte de 12 meses, e parte da diretoria de vendas, sendo consolidado com a diretoria de produção, onde é analisado se a fábrica tem capacidade de atender os pedidos de venda. Há também o planejamento de produção mensal. Deste plano de produção mensal, é extraído o programa de produção diária. A quantidade de produção diária é calculada pela média da quantidade total mensal.

Após o cálculo de um plano de produção mensal, o próximo passo é a programação de tarefas diárias, que especifica a ordem de montagem dos produtos finais. A montadora não utiliza nenhum método quantitativo para tomar decisões de programação. As decisões são tomadas em cima de métodos qualitativos, ou seja, de opiniões e consenso. Nesta etapa, introduziremos o uso do modelo, que determina a seqüência ótima de produção, de forma que a velocidade de retirada das peças de cada processo seja constante. A sequiência é medida em função do tempo de ciclo de um produto. Para determinar a programação de tarefas diária, será necessária a quantidade média de produção diária de cada produto e a quantidade de peças que compõe cada produto.

A montagem final compreende os seguintes produtos: eixo leve, eixo médio e eixo pesado. A demanda mensal de cada tipo de produto e a quantidade média diária de produção está na Tabela 1.

A programação de tarefas é comunicada somente para a linha de montagem final. Os processos precedentes não necessitam da programação antecipada. As funções do kanban dão as ordens de produção para o início do processo precedente, passo a passo.

Para adaptar-se às variações de demanda, a empresa trabalha com um sistema de folga e transferências de operários entre linhas. No sistema de folga, existe um banco de horas, onde o empregado pode ter até 100 horas positivas ou negativas. $\mathrm{O}$ uso de hora extra e subcontratação são bastante limitados. Procura-se manter constante o número de operários, evitando contratação e demissão de pessoal. 


\begin{tabular}{|c|c|c|c|}
\hline Tipos & Demanda mensal & $\begin{array}{c}\text { Média diária } \\
\text { produção }\end{array}$ & Tempo de Ciclo \\
\hline$A_{1}$ & $940 \mathrm{U}$ & $47 \mathrm{U}$ & $=(480 \mathrm{MIN} \mathrm{X}$ \\
\hline$A_{2}$ & $540 \mathrm{U}$ & $27 \mathrm{U}$ & $2 \mathrm{TURNOS} /$ \\
\hline$A_{3}$ & $720 \mathrm{U}$ & $36 \mathrm{U}$ & $110 \mathrm{U})$ \\
\hline TOTAL & $2.200 \mathrm{U}$ & $110 \mathrm{U} / \mathrm{DIA}$ & $=8.73 \mathrm{MIN} / \mathrm{U}$ \\
\hline
\end{tabular}

Fonte: Indústria Automobilística (2004).

Tabela 1 - Demanda mensal de cada produto e média diária de produção

Legenda:

$A_{1}=$ eixos leves

$A_{2}=$ eixos médios

$A_{3}=$ eixos pesados

Portanto, em função da demanda mensal, calcula-se a quantidade média diária de produção de cada tipo de produto.

\begin{tabular}{|c|c|c|c|}
\hline Produtos $A_{i}$ & $A_{1}=$ eixos leves & $A_{2}=$ eixos médios & $A_{3}=$ eixos pesados \\
\hline $\begin{array}{c}\text { Quantidade de produção } \\
Q_{i}\end{array}$ & 47 & 27 & 36 \\
\hline
\end{tabular}

Fonte: Indústria automobilística (2004).

Tabela 2 - Quantidade média diária de produção de cada produto

A Tabela 3 mostra a quantidade de cada peça necessária para a produção dos três tipos de produtos.

\begin{tabular}{|c|c|c|c|c|c|c|c|}
\hline Peça & $a_{1}=$ viga & $a_{2}=$ manga & $a_{3}=$ cubo & $a_{4}=$ disco & $a_{5}=$ tambor & $a_{6}=$ freio & $a_{7}=$ caliper \\
\hline$A_{1}$ & 1 & 2 & 2 & 2 & 0 & 0 & 2 \\
\hline$A_{2}$ & 1 & 2 & 2 & 0 & 2 & 2 & 0 \\
\hline$A_{3}$ & 1 & 2 & 2 & 0 & 2 & 2 & 0 \\
\hline
\end{tabular}

Fonte: Indústria automobilística (2004).

Tabela 3 - Quantidade de cada peça para produção de cada produto

O objetivo da programação de tarefas para a linha de montagem é obter a sequência ótima do mix de produtos, numa velocidade e quantidade de retirada constante, em função do tempo de ciclo de cada peça. Neste sistema de "puxar" do kanban, a variação da quantidade média consumida de cada peça na linha de montagem final deve ser minimizada. Portanto, a quantidade consumida por hora ou a velocidade de consumo de cada peça na linha de montagem deve ser mantida constante. A 
determinação da sequência ótima do mix de produção permite retiradas niveladas dos processos de submontagens e suprimentos.

Os objetivos para controlar a Linha de Montagem são:

1. Nivelar o tempo total de montagem de cada processo da linha

2. Manter uma velocidade constante na montagem de cada peça da linha

\section{Modelagem matemática do problema}

A modelagem matemática do problema foi baseada nos trabalhos de JOHNSON e MONTGOMERY (1974), MONDEY (1981), MONDEY (1984) e WINSTON (1995).

Descreveremos abaixo os dois objetivos para controlar a linha de montagem.

1. Nivelar o tempo total de montagem de cada processo da linha

O tempo de operação de cada processo, estabelecido pelo mix de produção, não deve exceder o tempo do ciclo pré-determinado:

$$
\max _{j}\left\{\frac{\sum_{i=1}^{n} Q_{i} T_{i j}}{\sum_{i=1}^{n} Q_{i}}\right\} \leq C,
$$

onde:

$Q_{i}=$ Quantidade de produção do produto $A_{i}(i=1, \ldots, n)$

$T_{i j}=$ Tempode operação do produto $A_{i}$ no processo $j$

$C=$ Tempodo ciclo $=\frac{\text { Tempo total de operação por dia }}{\sum^{n} Q_{i}}$

$$
\sum_{i=1}^{n} Q_{i}
$$

Se os produtos com tempo de produção relativamente longo forem sucessivamente introduzidos na linha, terão uma demora para serem completados e poderão causar paradas de linha. Este primeiro objetivo é incorporado à seqüência algoritma do segundo objetivo.

2. Manter uma velocidade constante na montagem de cada peça da linha e determinar a seqüência do modelo

A velocidade de consumo ou retirada de cada peça da linha deve ser constante. As variáveis do modelo são:

$Q=$ Quantidade total de produção de todos os produtos $A_{i}(i=1, \ldots, n)$ 
$Q=\sum_{i=1}^{n} Q_{i},\left(Q_{i}=\right.$ quantidade de produção de cada produto $\left.A_{i}\right)$

$N_{j}=$ Quantidade total necessária da peça $a_{j}(j=1, \ldots, m)$ a ser consumida para a produção de todos os produtos $A_{i}(i=1, \ldots, n)$.

$X_{j k}=$ Quantidade total necessária da peça $a_{j}$ a ser utilizada para produzir os produtos da seqüência determinada do primeiro ao $k$-ésimo produto.

$\frac{N_{j}}{Q}=$ Quantidade média necessária da peça $a_{j}$ por unidade de produto.

$\frac{k \cdot N_{j}}{Q}=$ Quantidade média necessária da peça $a_{j}$ para produzir $k$ unidades de produtos.

A fim de manter a velocidade de consumo constante de cada peça $a_{j}$, o montante de $X_{j k}$ deve ser o mais próximo possível do valor de $\frac{k \cdot N_{j}}{Q}$.

$$
\min D_{k, i}=\sqrt{\sum_{j=1}^{m}\left(\frac{k \cdot N_{j}}{Q}-X_{j k}\right)^{2}}
$$

ou,

$$
\min D_{k, i}=\sqrt{\sum_{j=1}^{m}\left(\frac{k \cdot N_{j}}{Q}-X_{j, k-1}-b_{i j}\right)^{2}}
$$

onde:

$b_{i j}=$ quantidade necessária da peça $a_{j}(j=1, \ldots, m)$ para produzir uma unidade do produto $A_{i}(i=1, \ldots, n)$.

\section{APLICAÇÃO DO MODELO E RESULTADOS}

De acordo com as Tabelas 2 e 3, determinaremos a seqüência ótima de produção.

Primeiramente será incorporado o objetivo 1 à solução algoritma do segundo objetivo. Além do número de peças de cada produto, é importante considerar o tempo total de montagem de cada uma das sete peças, para os três produtos. O tempo de montagem da viga, cubo, disco, freio e caliper são iguais para os três produtos. Portanto, para estas peças, os valores da Tabela 3 permanecem inalterados. Já para a manga, o tempo de montagem do eixo médio é $20 \%$ superior ao eixo leve, e do eixo pesado é $50 \%$ maior que o eixo leve, enquanto que no tambor, o tempo de montagem do eixo médio é $30 \%$ superior ao eixo leve, e do eixo pesado é $80 \%$ maior que o eixo 
leve. Os novos valores da Tabela 3, considerando agora os tempos de montagens, encontram-se na Tabela 4.

A quantidade total necessária $\left(N_{j}\right)$ da peça $a_{j}(j=1, \ldots, 7)$ para produzir todos os produtos $A_{i}(i=1, \ldots, n)$ é:

$$
N_{j}=Q_{i} \cdot b_{i j}
$$

$$
N_{j}=[47,27,36]\left[\begin{array}{ccccccc}
1 & 2 & 2 & 2 & 0 & 0 & 2 \\
1 & 2,4 & 2 & 0 & 2,6 & 2 & 0 \\
1 & 3 & 2 & 0 & 3,6 & 2 & 0
\end{array}\right]=\left[\begin{array}{c}
110 \\
267 \\
220 \\
094 \\
200 \\
126 \\
094
\end{array}\right]
$$

\begin{tabular}{|c|c|c|c|c|c|c|c|}
\hline Peça & $a_{1}=\mathbf{v i g a}$ & $a_{2}=$ manga & $a_{3}=$ cubo & $a_{4}=$ disco & $a_{5}$ =tambor & $a_{6}=$ freio & $a_{7}=$ caliper \\
\hline$A_{1}$ & 1 & 2 & 2 & 2 & 0 & 0 & 2 \\
\hline$A_{2}$ & 1 & 2,4 & 2 & 0 & 2,6 & 2 & 0 \\
\hline$A_{3}$ & 1 & 3 & 2 & 0 & 3,6 & 2 & 0 \\
\hline
\end{tabular}

Fonte: Indústria automobilística (2004).

Tabela 4 - Quantidade necessária de cada peça para produção dos três produtos considerando tempo de montagem

Legenda:

$A_{1}=$ eixos leves

$A_{2}=$ eixos médios

$A_{3}=$ eixos pesados

A quantidade total de produção de todos os produtos $A_{i}(i=1,2,3)$ é:

$Q=\sum_{i=1}^{3} Q_{i}=47+27+36=110$

Portanto:

$\frac{N_{j}}{Q}=[110 / 110,267 / 110,220 / 110,94 / 110,200 / 110,126 / 110,94 / 110],(j=1, \ldots, 7)$

$\frac{N_{j}}{Q}=[1,2.43,2,0.85,1.82,1.15,0.85],(j=1, \ldots, 7)$

$k=1, \ldots, 110$

Quando $k=1$

Para $A_{1}=$ 


$$
\begin{aligned}
& \sqrt{(1 \cdot 1-0-1)^{2}+(1 \cdot 2,43-0-2)^{2}+(1 \cdot 2-0-2)^{2}+(1 \cdot 0,85-0-2)^{2}} \\
& +(1 \cdot 1,82-0-0)^{2}+(1 \cdot 1,15-0-0)^{2}+(1 \cdot 0,85-0-2)^{2} \\
& D_{1,1}=2.73
\end{aligned}
$$

Para $A_{2}=$

$$
\begin{aligned}
& \sqrt{\begin{array}{l}
(1 \cdot 1-0-1)^{2}+(1 \cdot 2,43-0-2,4)^{2}+(1 \cdot 2-0-2)^{2}+(1 \cdot 0,85-0-0)^{2} \\
+(1 \cdot 1,82-0-2,6)^{2}+(1 \cdot 1,15-0-2)^{2}+(1 \cdot 0,85-0-0)^{2}
\end{array}} \\
& D_{1,2}=1.67
\end{aligned}
$$

Para $A_{3}=$

$$
\begin{aligned}
& \sqrt{\begin{array}{l}
(1 \cdot 1-0-1)^{2}+(1 \cdot 2,43-0-3)^{2}+(1 \cdot 2-0-2)^{2}+(1 \cdot 0,85-0-0)^{2} \\
+(1 \cdot 1,82-0-3,6)^{2}+(1 \cdot 1,15-0-2)^{2}+(1 \cdot 0,85-0-0)^{2}
\end{array}} \\
& D_{1,3}=2.38 \\
& D_{1, \mathrm{i}}=\min (2.73,1.67,2.38)=1.67
\end{aligned}
$$

Portanto a primeira ordem da sequência é o produto $A_{2}$.

\section{Quando $k=2$}

Para $A_{1}=$

$$
\begin{aligned}
& \sqrt{(2 \cdot 1-1-1)^{2}+(2 \cdot 2,43-2,4-2)^{2}+(2 \cdot 2-2-2)^{2}+(2 \cdot 0,85-0-2)^{2}} \\
& +(2 \cdot 1,82-2,6-0)^{2}+(2 \cdot 1,15-2-0)^{2}+(2 \cdot 0,85-0-2)^{2} \\
& D_{2,1}=1.25
\end{aligned}
$$

Para $A_{2}=$

$$
\begin{aligned}
& \sqrt{(2 \cdot 1-1-1)^{2}+(2 \cdot 2,43-2,4-2,4)^{2}+(2 \cdot 2-2-2)^{2}+(2 \cdot 0,85-0-0)^{2}} \\
& \sqrt{+}(2 \cdot 1,82-2,6-2,6)^{2}+(2 \cdot 1,15-2-2)^{2}+(2 \cdot 0,85-0-0)^{2} \\
& D_{2,2}=3.33
\end{aligned}
$$

Para $A_{3}=$

$$
\begin{aligned}
& \sqrt{\begin{array}{l}
(2 \cdot 1-1-1)^{2}+(2 \cdot 2,43-2,4-3)^{2}+(2 \cdot 2-2-2)^{2}+(2 \cdot 0,85-0-0)^{2} \\
+(2 \cdot 1,82-2,6-3,6)^{2}+(2 \cdot 1,15-2-2)^{2}+(2 \cdot 0,85-0-0)^{2}
\end{array}} \\
& D_{2,3}=3.94 \\
& D_{2, \mathrm{i}}=\min (1.25,3.33,3.94)=1.25
\end{aligned}
$$

Portanto a segunda ordem na seqüência do programa é o produto $A_{1}$.

O mesmo raciocínio vale até $k=110$.

Para calcular a seqüência ótima do mix de produção foi desenvolvido um algoritmo em VBA, que fornece simultaneamente todo o programa ótimo de produção. Os detalhes do algoritmo de otimização estão em anexo. 
A seqüência ótima do programa de produção de $k=1, \ldots, 110$ é:

$\begin{array}{llllllllllllllllllllll}2 & 1 & 2 & 1 & 3 & 2 & 1 & 3 & 1 & 3 & 1 & 3 & 1 & 3 & 2 & 1 & 2 & 1 & 3 & 2 & 1 & 2 \\ 1 & 3 & 1 & 3 & 2 & 1 & 3 & 1 & 3 & 1 & 3 & 1 & 3 & 2 & 1 & 2 & 1 & 3 & 2 & 1 & 2 & 1 \\ 3 & 2 & 1 & 3 & 1 & 3 & 1 & 3 & 1 & 3 & 2 & 1 & 2 & 1 & 3 & 1 & 3 & 2 & 1 & 2 & 1 & 3 \\ 2 & 1 & 3 & 1 & 3 & 1 & 2 & 2 & 1 & 3 & 1 & 3 & 1 & 3 & 1 & 3 & 2 & 1 & 2 & 1 & 3 & 2 \\ 1 & 3 & 1 & 2 & 1 & 3 & 2 & 1 & 3 & 1 & 3 & 1 & 3 & 1 & 3 & 2 & 1 & 2 & 1 & 3 & 2 & 1\end{array}$

onde:

$A_{1}=1$

$A_{2}=2$

$A_{3}=3$

\section{Considerações finais}

O objetivo deste trabalho é determinar a sequiência ótima de produção, de forma que a velocidade de retirada de cada peça dos processos de submontagens seja constante, evitando assim a introdução sucessiva do mesmo produto que levaria a um longo tempo de operação. $\mathrm{O}$ modelo apresentado pode ser facilmente implementado em outras empresas que trabalham com produção puxada e produzem Just-in-time. A produção diária é em função de uma variedade de produtos e em pequenos lotes. Mantendo constante a velocidade de consumo das peças de cada processo de submontagem, o nível de estoques em processo e de produtos acabados são reduzidos ou mantidos constantes, as variações de demanda são adaptadas facilmente, e evita-se paradas de linha.

A aplicação adequada do modelo leva a empresa a obter maiores lucros e melhor retorno sobre o capital investido, decorrente da redução de custos e redução de estoques.

Como proposta de melhoria, poderia ser incorporado ao modelo, algumas restrições adicionais, tais como capacidade dos equipamentos, etc.

\section{ABSTRACT}

The aim of this paper is to determine the optimized production sequence for a product mix in order to allow regular withdraws of the sub-assembly processes and supplies. This model is applied in Just in time (JIT) pull production systems. In this system, the daily production is varied and on low scale. Therefore, it can easily adapt to demand variation and reduce inventory. In applying this model the business can obtain more profit and better return on the invested capital due to cost reduction, inventory reduction and quality improvement.

Key words: Scheduling, Just in time, Pull production.

\section{Referências}

BUFFA, E. S.; SARIN, R. K. Modern production/operations management. 8. ed. New York : Wiley, 1987.

CHASE, R. B.; AQUILANO, N. J.; JACOBS, F. R. Production and operations management. 8. ed. Boston : McGraw-Hill, 1998.

CROSBY, L. B. The Just-In-Time Manufacturing Process. Production and inventory management, v. 25, n. 4, p. $110-122,1984$.

JOHNSON, A. J.; MONTGOMERY, D. C. Operations research in production planning, scheduling, and inventory control. New York : Wiley, 1974. 
MONDEN, Y. Smoothed production lets toyota adapt to demand changes and reduce inventory. Industrial Engineering, v. 13, n. 8, p. 42-51, 1981.

MONDEN, Y. Sistema Toyota de produção. São Paulo : Imam, 1984.

WINSTON, W. L. Introduction to mathematical programming: applications and algorithms. 2. ed. Duxbury Press, 1995.

\section{ANEXO}

Algoritimo de otimização

Programa Principal

Option Explicit

Option Base 1

Public Num_Produtos As Integer, K As Integer, Num_peças As Double,

Tot_Produção As Integer, Desvio(200) As Double, Ordem(200) As Integer,

B(200, 100) As Double, XJ(200) As Double, Peso(200) As Double

Sub Principal( )

Call Inicializa

For K = 1 To Tot_Produção

Call Gera_Sequencia

Call Le_Somatoria

Call Le_Desvio

Call Escolhe_Min

Next K

End Sub

Rotinas

Option Explicit

Option Base 1

Sub Inicializa( )

Inicializa Total produzido, número de produtos, número de peças

e zera somatória

Dim I As Integer, IB As Integer, JB As Integer, IP As Integer

With Range("B1")

Tot_Produção $=$.Offset $(1,0)$

End With

With Range("B7")

Num_Produtos $=$ Range $($. Offset $(0,0)$, .End(xlDown) $)$.Rows.Count

End With

With Range("C1")

Num_peças $=$ Range $($. Offset $(0,0)$, .End(xlToRight $))$. Columns.Count

Num_peças = Num_peças -1 'O peso não faz parte da estrutura.

End With

$\mathrm{K}=0$

' Zera Somatória e K

For I = 1 To Num_peças

With Range("B6")

.Offset $(0, \mathrm{I})=0$ 
End With

Next

With Range("B5")

.Offset $(0,0)=0$

End With

With Range("J7")
For IP = 1 To Num_Produtos
Peso(IP) = .Offset(IP - 1,0)
Next
End With

With Range("B6")

For IB = 1 To Num_Produtos

For JB = 1 To Num_peças

$\mathrm{B}(\mathrm{IB}, \mathrm{JB})=\operatorname{Peso}(\mathrm{IB}) *($. Offset $(\mathrm{IB}, \mathrm{JB}))$

Next JB

Next IB

End With

With Range("B11")

For IB = 1 To Num_Produtos

For JB $=1$ To Num_peças

.Offset $(\mathrm{IB}, \mathrm{JB})=\mathrm{B}(\mathrm{IB}, \mathrm{JB})$

Next JB

Next IB

End With

End Sub

Sub Gera_Sequencia( )

With Range("B5")

.Offset $(0,0)=K$

End With

End Sub

Sub Le_Somatoria( )

Dim IC As Integer

' Lê quantidade necessária anterior da peca ai

With Range("C6")

For IC = 1 To Num_peças

$\mathrm{XJ}(\mathrm{IC})=. \operatorname{Offset}(0, \mathrm{IC}-1)$

Next

End With

End Sub

Sub Le_Desvio( )

Dim ID As Integer

With Range("R7")

For ID = 1 To Num_Produtos 


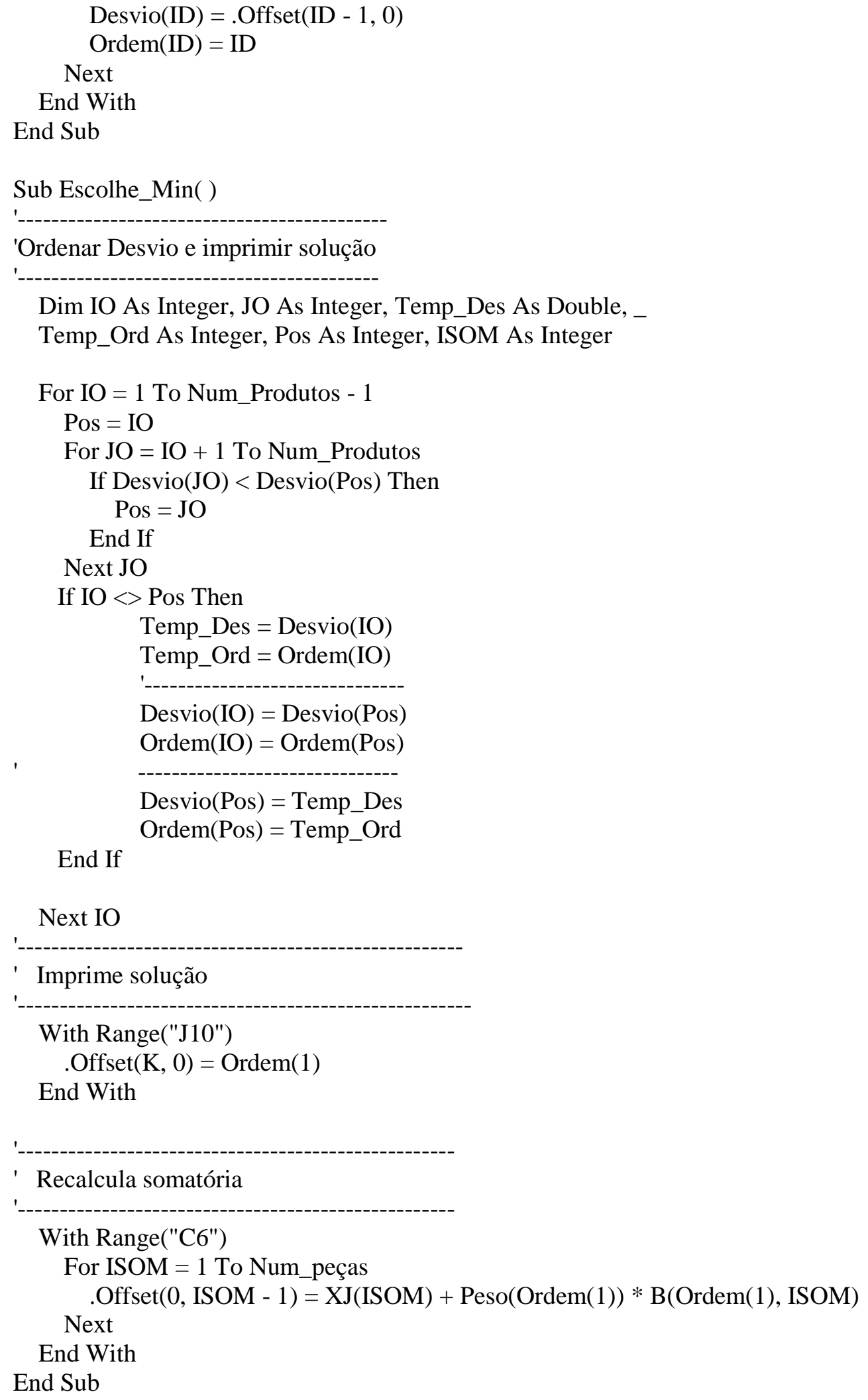

Next IO

' Imprime solução

With Range("J10")

.Offset $(\mathrm{K}, 0)=\operatorname{Ordem}(1)$

End With 